\title{
Beitrag zur Dimensionierung von automatischen Sortiersystemen (Sorter)
}

\author{
DiPL.-ING. KARSTEN TUREK \\ PROF. DR.-ING. HABIL. HANS-GEORG MARQUARDT \\ INSTITUT FÜR FÖRDERTECHNIK, BAUMASCHINEN UND LOGISTIK, TECHNISCHE UNIVERSITÄT DRESDEN
}

\section{Zusammenfassung}

Automatische Sortiersysteme (Sorter) besitzen in der Intralogistik eine große Bedeutung. Sorter erreichen eine ausdauernd hohe Sortierleistung bei gleichzeitig geringer Fehlsortierrate und bilden deshalb oft den zentralen Baustein in Materialflusssystemen mit hoher Umschlagsrate. Distributionszentren mit Lager und Kommissionierfunktion sind typische Vertreter solcher Materialflusssysteme. Ein Sorter besteht aus den Subsystemen Einschleusung, Verteilförderer und Endstellen. Die folgenden Betrachtungen fokussieren auf ein Sortermodell mit einem Verteilförderer in Ringstruktur und einer Einzelplatzbelegung. Auf jedem Platz kann genau ein Gut transportiert werden. Der Verteilförderer besitzt somit eine feste Transportkapazität. Derartige Förderer werden in der Regel als Kippschalen- oder Quergurt-Sorter ausgeführt. Die theoretische Sortierleistung für diesen Sortertyp kann aus Fahrgeschwindigkeit und Transportplatzabstand bestimmt werden. Diese Systemleistung wird im praktischen Betrieb kaum erreicht. Verschiedene Faktoren im Einschleusbereich und im Ausschleusbereich führen zu einer Leistungsminderung. Betrachtungen zur Bestimmung der mittleren Warteschlangenlänge im Einschleusbereich sowie zur Ermittlung des Rundläuferanteils auf dem Verteilförderer werden im folgenden Beitrag vorgestellt. Diesem Beitrag liegt ein Forschungsvorhaben zugrunde, das aus Mitteln des Bundesministeriums für Wirtschaft und Technologie (BMWi) über die Arbeitsgemeinschaft industrieller Forschungsvereinigungen "Otto von Guericke" (AiF) gefördert und im Auftrage der Bundesvereinigung Logistik e.V. (BVL) ausgeführt wurde.

\section{Abstract}

Fully automated sortation systems (sorter) gain increasing relevance for the intra logistic sector. Sorter achieve a constant high sortation performance accompanied by low sortation error rate, and on that score often they are the backbone of complex distribution systems. A sorter system consits of inductions, transport conveyor, and destinations. The following article focuses on a sorter model with a closed loop transport conveyor and with single tray allocation on it. The transport conveyor has a fixed transport capacity. These conveyors are mostly realised in tilt tray or belt tray technology. The theoretical sorting performance, calculated by transport speed and tray distance, can merely achieved in industrial application. A set of factors cause a loss of performance. The following article presents a method to calculate the average waiting queue at inductions and the loss of performance by circulating goods on transport conveyor. The calculation is based on simulation experiments and polynomical approximation. This paper is based on a research project which was funded by the Federal Ministry of Economics and Technology via the Arbeitsgemeinschaft industrieller Forschungsvereinigungen "Otto von Guericke" (AiF) and carried out on behalf of the Bundesvereinigung Logistik e.V. (BVL).

\section{Einführung}

Die Bestimmung der notwendigen Warteraumgröße bei bestimmter Systembelastung ist ein kritisches Problem bei der Planung von Materialflusssystemen. Die Entscheidung über die richtige Dimensionierung des Systems kann oft nicht als binäre Aussage gegeben werden, sondern sie hängt von den auftretenden und akzeptierbaren Warteschlangenlängen ab. Die Modellierung mit der Bedienungstheorie kann einen analytischen Zugang für die Ermittlung dieser Systemkenngrößen bieten, setzt aber oft spezielle Bedingungen für die Modellgültigkeit voraus. Weitere Erkenntnisse können dann nur mit Hilfe spezifischer Simulationsmodelle gewonnen werden. Einen dritten Weg bieten Näherungsverfahren auf der Basis von Parametergleichungen. Die Ausgangsdaten werden dabei entweder aus dem realen Betrieb des Systems oder über ein Simulationsmodell gewonnen. Für die Datenauswertung und die Parameterbestimmung stehen verschiedene Verfahren zur Verfügung. Die Näherung über Polynome wird in [Schulze2000] als geeigneter heuristischer Ansatz beschrieben. 
Polynome sind in der Lage, stetige Kenngrößenzusammenhänge abzubilden. Eine Näherungslösung zur Bestimmung der mittleren Warteschlangenlänge im Einschleusbereich eines Sorters sowie zur Ermittlung des Rundläuferanteils auf dem Verteilförderer wird vorgestellt.

\section{Einschleusbereich}

Insbesondere bei Hochleistungssortern genügt eine Einschleuslinie nicht für die Versorgung des Verteilförderers. Übliche Leistungsgrenzen von Einschleusungen werden in [Hirsch2004] mit 3000 - 6000 Stück pro Stunde angegeben. Zur Steigerung der Systemleistung werden deshalb mehrere Einschleuslinien parallel angeordnet. Sie bilden zusammen einen Einschleusbereich. Der Einschleusbereich ist in der Regel so dimensioniert, dass alle Transportplätze auf dem Verteilförderer erreicht werden können. Für die Güter auf einer Einschleuslinie ergibt sich ein technisch bedingter Abstand zwischen zwei Einschleusungen - der Einschleusabstand.

Die parallelen Einschleuslinien beeinflussen sich aber gegenseitig in ihrer Leistung, da sie gleichzeitig auf den Bestand an freien Transportplätzen zugreifen. Das Problem einer Mehrfachbelegung eines Transportplatzes wird dadurch gelöst, dass vor der Durchführung des technischen Einschleusvorganges die Einschleusung einen freien Transportplatz reservieren muss.

Die parallelen Einschleuslinien konkurrieren dabei mit ihren Reservierungsanforderungen. Im Interesse einer optimalen Einschleusung und einer maximalen Auslastung der gegebenen Transportkapazität ist ein Ausgleich zwischen den Einschleuslinien erforderlich. Der Ausgleich erfolgt durch eine gesteuerte Zuweisung der freien Plätze an die Einschleuslinie, durch ein Leerplatz-Zuweisungsverfahren. Für die Realisierung der Zuweisung existieren verschiedene Strategien:

- $\quad$ Stochastische Zuweisung [JoHo2006]:

Die Zuweisung erfolgt in der Reihenfolge der Ankunft (FCFS) an den Einschleusungen.

- $\quad$ Statische [Beumer1993] oder serielle [JoHo2006] Zuweisung:

Die Zuweisung für jede Einschleusung erfolgt im konstanten Anteil verfügbarer Transportplätze.

Die Anteile beruhen auf den Lastannahmen für die Einschleusungen.

- Dynamische [Beumer1993] oder flexible [JoHo2006] Zuweisung:

Die Zuweisung erfolgt in variablen Anteilen verfügbarer Transportplätze auf der Basis einer Priorität. Die Priorität wird dynamisch aus den aktuellen Lastannahmen (Lastanteil zur Gesamtlast) und dem Arbeitsstatus (aktiv oder ruhend) bestimmt.

- $\quad$ Referenzpunktverfahren [Beumer1993]:

Auf dem Verteilförderer existiert für alle Einschleusungen ein zentraler Zuweisungspunkt. Die Bedarfsermittlung erfolgt an einem vorgelagerten Referenzpunkt auf der Einschleuslinie.

Von diesen Verfahren garantiert das Referenzpunktverfahren im Einschleusbereich die geringsten Verluste an freier Transportkapazität. Das Referenzpunktverfahren wurde deshalb als Zuweisungsverfahren im Untersuchungsmodell implementiert.

\subsection{Modellierung als $\mathbf{M} / \mathbf{D}^{\prime} / 1-$ System}

Das M/D'/1-Modell, vorgestellt in [MeiSun2007], entsteht durch eine Modifikation des M/D/1-Modells im Bedienprozess. Die Bedienstation arbeitet in beiden Modellen mit einer konstanten Bedienzeit. Im M/D/1Modell beginnt die Bearbeitung einer Forderung bei leerer Bedienstation unmittelbar nach Forderungsankunft im System oder nach Bedientaktende bei übrigen Forderungen im Warteraum. Im M/D'/1-Modell beginnt die Bearbeitung von Forderungen immer in einem konstanten Zeitabstand, unabhängig vom Zustand der Bedienstation. Eine Forderung, die auf ein leeres System trifft, muss auf den Beginn des nächsten Bedientaktes warten.

Die Leerplatz-Zuweisung nach dem Referenzpunktverfahren im Einschleusbereich eines Sorters kann ebenfalls als M/D'/1-System modelliert werden, wenn exponentialverteilte Zwischenankunftszeiten im Ankunftsprozess angenommen werden. Die Einzelplätze auf dem Sorter besitzen alle die gleiche Länge, die Güter auf dem Sorter damit alle den gleichen Mindestabstand. Das Gut, welches am Referenzpunkt der Einschleusung eintrifft, muss mindestens auf das Vorbeifahren des nächsten Einzelplatzes am zentralen Zuweisungspunkt warten, bevor die Leerplatz-Zuweisung erfolgen kann. Im Einschleusbereich entsteht zwischen zwei aufeinander folgenden Zuweisungen ein Mindestabstand, der sich aus der Teilung und der Fahrgeschwindigkeit des Sorters ergibt. 
Auf der Basis dieser Überlegungen lässt sich die mittlere Gesamt-Warteschlange der Güter im Einschleusbereich in Abhängigkeit von der Belastung nach dem Ansatz für das M/D'/1-Modell berechnen.

$$
E\left[N_{w, M / D^{\prime} / 1}\right]=\frac{\rho}{2(1-\rho)}
$$

Die so ermittelte Warteschlange gilt für den Einschleusbereich als ganzes. Diese Warteschlange teilt sich dann auf die einzelnen Einschleuslinien entsprechend ihrer Anteile an der Gesamtlast auf. In Simulationsexperimenten konnte dieser Zusammenhang bestätigt werden.

Dieser Berechnungsansatz nach Bedientheorie liefert korrekte Werte nur für Einschleuslinien ohne Einschleusabstand. In der Praxis liegt zwischen zwei aufeinander folgenden Einschleusvorgängen ein Mindestzeitabstand. Eine Einschleuslinie kann nicht alle Transportplätze auf dem Verteilförderer erreichen. Bei gleicher Einschleuslast wächst die Warteschlange mit zunehmendem Einschleusabstand. Die Aufteilung der Warteschlange auf die Einschleuslinien entsprechend der Anteile an der Gesamtlast gilt nicht mehr.

Da die analytische Modellierung mit der Bedientheorie hier an Grenzen stößt, ergab sich die Notwendigkeit für einen anderen Zugang für die Bestimmung der mittleren Warteschlange an den Einschleuslinien.

\subsection{Bestimmung der Warteschlangenlänge durch Näherung}

Einen alternativen Weg für die Bestimmung der Kenngrößenzusammenhänge bietet das Näherungsverfahren auf der Basis von Polynomgleichungen. Die Näherung über Polynome wird in [Schulze2000] als geeigneter heuristischer Ansatz beschrieben, um Kennfelder von Materialflusskomponenten und Materialflusssysteme zu beschreiben. Die Polynome sind in der Lage, beliebige stetige Kenngrößenzusammenhänge abzubilden. Die Exponenten der Polynome werden in einem Optimierungsverfahren mit Genetischem Algorithmus ermittelt. Die Anpassung der Koeffizienten erfolgt nach der Methode der kleinsten Abstandsquadrate. Das in [Schulze2000] vorgestellte Verfahren Approx liefert für die Annäherung an einen beliebigen Datensatz mit N Kenngrößen im Ergebnis ein Polynom mit K Gliedern in der Form

$$
Z=\mathrm{p}(\boldsymbol{x})=\sum_{k=1}^{K} c_{k} \prod_{n=1}^{N} x_{n}^{e_{k, n}}
$$

Für mehrere parallele Einschleuslinien in einem Einschleusbereich soll für verschiedene Einschleusabstände und Lastverteilungen der effektive Auslastungswert $\rho_{\mathrm{E}}$ ermittelt werden, aus dem über die oben genannte Formel (1) die jeweilige mittlere Warteschlange der Linie berechnet werden kann. Die Erhöhung des Einschleusabstandes führt zu einer Erhöhung der mittleren Bedienzeit und damit zu einer höheren Auslastung der Einschleuslinie.

Für die Datengewinnung wurden systematische Simulationsexperimente in einem Parameterraum durchgeführt. Der Einschleusabstand wurde zwischen 2, 3 und 4 verändert. Die Gesamtauslastung im Einschleusbereich wurde schrittweise von 33\% bis 95\% erhöht, soweit dies für die einzelne Einschleuslinie möglich war. Für den Einschleusbereich wurden verschiedene Konfiguration mit 3, 4 und 5 Einschleuslinien mit unterschiedlichen spezifischen Lastanteilen gewählt. Das Ziel der Datenauswertung bestand darin, den Zusammenhang zwischen der veränderten Auslastung $\rho_{\mathrm{E}}$ als Folge von Einschleusabstand, Gesamtauslastung und Lastanteil aufzuzeigen und als Näherungspolynom zu beschreiben.

Bei der Bestimmung des Näherungspolynoms wurde die Anzahl der Polynomglieder und der Grad der Exponenten in mehreren Testläufen manuell verändert mit dem Ziel, bei möglichst geringer Anzahl und Grad eine hinreichende Genauigkeit der Näherung zu erzielen. Als Ergebnis wurden als Näherungen Polynome mit 6 Gliedern und Exponenten 2. Grades gewählt. Die Näherungslösung gilt jedoch nur für KenngrößenKonfigurationen innerhalb des untersuchten Parameterraumes.

Tabelle 1:Kenngrößen im Näherungspolynom für $\rho_{E}$

\begin{tabular}{cc}
\hline Index & Kenngröße \\
\hline 1 & Auslastung des Einschleusbereichs $\rho_{\mathrm{B}}$ \\
2 & Anteil der Einschleuslinie $\mathrm{p}_{\mathrm{E}}$ \\
\hline
\end{tabular}

Das Programm Approx lieferte in Auswertung der Experimente mit Einschleusabstand 2 das folgende Nährungspolynom mit der Struktur entsprechend (2):

$$
\rho_{E}=-2,645 \cdot \rho_{B}^{2} \cdot p_{E}^{2}-0,209 \cdot \rho_{B}^{2} \cdot p_{E}+3,561 \cdot \rho_{B} \cdot p_{E}^{2}+0,960 \cdot \rho_{B}-0,584 \cdot p_{E}^{2}+0,05355
$$


Nachfolgend werden die Koeffizienten und Exponenten der Nährungspolynome für die betrachteten Einschleusabstände ( $\mathrm{e}=2$, e $=3$ und e = 4) kompakt in tabellarischer Form dargestellt. Aus dem Vergleich der eben genannten Formel mit Tabelle 2 erschließt sich die Tabelleninterpretation auf einfache Weise.

Tabelle 2:Näherung für Einschleusabstand 2

\begin{tabular}{lrrr}
\hline$k$ & $c_{k}$ & $e_{k, 1}$ & $e_{k, 2}$ \\
\hline 1 & -2.6448683576 & 2 & 2 \\
2 & -0.2092760632 & 2 & 1 \\
3 & 3.5610338725 & 1 & 2 \\
4 & 0.9598724759 & 1 & 0 \\
5 & -0.5842464894 & 0 & 2 \\
6 & 0.0535499420 & 0 & 0 \\
\hline
\end{tabular}

Tabelle 3:Näherung für Einschleusabstand 3

\begin{tabular}{rrrr}
\hline$k$ & $c_{k}$ & $e_{k, 1}$ & $e_{k, 2}$ \\
\hline 1 & -2.6853443427 & 2 & 1 \\
2 & 2.1227058600 & 1 & 2 \\
3 & 2.0353857380 & 1 & 1 \\
4 & 1.0141942496 & 1 & 0 \\
5 & -0.4753509157 & 0 & 2 \\
6 & 0.0113760562 & 0 & 0 \\
\hline
\end{tabular}

Tabelle 4: Näherung für Einschleusabstand 4

\begin{tabular}{lrrr}
\hline$k$ & $c_{k}$ & $e_{k, 1}$ & $e_{k, 2}$ \\
\hline 1 & 4.9553943844 & 2 & 2 \\
2 & -4.6477225234 & 2 & 1 \\
3 & -2.1970085167 & 1 & 2 \\
4 & 3.5086455768 & 1 & 1 \\
5 & 1.0140438730 & 1 & 0 \\
6 & 0.3310409340 & 0 & 1 \\
\hline
\end{tabular}

Der Vergleich von Datensatz und Näherung zeigt in weiten Bereichen eine recht gute Übereinstimmung der Polynompunkte mit den Datenpunkten. Der maximale relative Fehler vergleicht die Näherungswerte für $\rho_{\mathrm{E}}$ mit den Simulationswerten und gibt den maximalen Wert des relativen Fehlers über alle Datenpunkte an. Der maximale absolute Fehler gibt die größte Differenz für die mittlere Warteschlange zwischen Simulation und Näherung an. Da die größten Warteschlangen bei Einschleusabstand e $=4$ auftreten, ist hier trotz des geringeren relativen Fehlers der absolute Fehler am größten.

Tabelle 5:Vergleich der Näherungen für Einschleusabstände

\begin{tabular}{ccc}
\hline$e$ & $\begin{array}{c}\text { max. rel. } \\
\text { Fehler }\end{array}$ & $\begin{array}{c}\text { max. abs. } \\
\text { Fehler }\end{array}$ \\
\hline 2 & $2,0 \%$ & 3,0 St. \\
3 & $2,2 \%$ & 2,8 St. \\
4 & $1,6 \%$ & 4,2 St. \\
\hline
\end{tabular}


Die Überblendung von Datensatz und Näherung für e = 2 in Abbildung 1 zeigt eine recht gute Übereinstimmung der Polynompunkte mit den Datenpunkten. Die nach Formel (1) berechneten Werte für die Warteschlangenlänge sind als Gitter im Diagramm hinterlegt. Die grafischen Vergleiche der anderen vorgestellten Näherungen ergeben ein qualitativ ähnliches Bild.

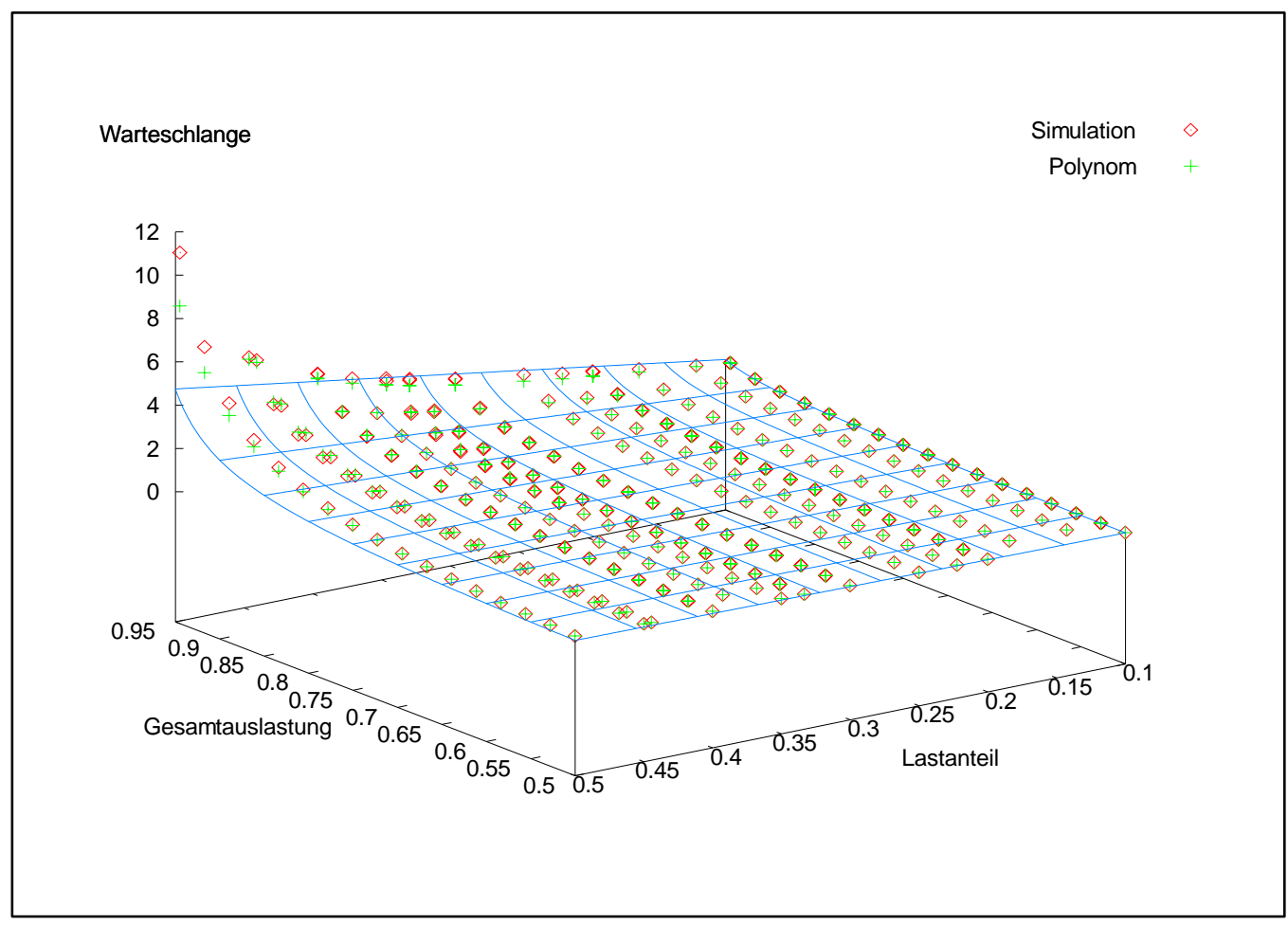

Abbildung 1: Vergleich der Interpolation für e = 2

Die ermittelten Polynomgleichungen liefern eine Näherung für den beschriebenen Kenngrößenzusammenhang im Einschleusbereich unter den genannten Voraussetzungen und für den durch die Experimentdaten abgegrenzten Parameterraum.

Die Entscheidung über die praktische Belastungsgrenze des Sorters hängt nun von den im Einschleusbereich auftretenden Warteschlangenlängen ab, die im konkreten Einsatzfall akzeptiert werden können. Damit können Aussagen zur Dimensionierung sowie zur maximal zulässigen Belastung des Einschleusbereichs gegeben werden.

\section{Ausschleusbereich}

Leistungsverluste im Ausschleusbereich entstehen dann, wenn Güter den Verteilförderer nicht an den Endstellen verlassen, sondern auf dem Sorter verbleiben. Aufgrund der Ringstruktur des Verteilförderers behindern diese zirkulierenden Güter durch die Belegung von Transportplätzen das Einschleusen neuer Güter. Die Transportkapazität wird nicht effizient genutzt.

Im praktischen Betrieb erfordert der Ausschleusprozess eine bestimmte Zeitspanne. Die Sensoren an der Endstelle müssen die korrekte Abförderung des Gutes bestätigen, bevor das nächste Gut über die gleiche Endstelle den Verteilförderer verlassen kann. Die notwendige Zeitspanne hängt von der technischen Realisierung der Ausschleusung ab. Zwischen zwei aufeinander folgenden Gutausschleusungen entsteht somit ein Mindestzeitabstand, nachfolgend als Ausschleusabstand bezeichnet. Die Ausschleusung kann dann nicht alle Transportgüter in unmittelbarer Folge erreichen, wenn der Ausschleusabstand größer ist als der Einzelplatzabstand auf dem Verteilförderer.

Transportgüter, die aus diesem Grund auf dem Verteilförderer verbleiben, werden als Rundläufer bezeichnet, da sie in der Regel eine weitere Runde auf dem Verteilförderer transportiert werden, bis sie erneut ihre Endstelle erreichen.

Nachfolgend wird für diese Rundläufer ein Ansatz für die Abschätzung des Anteils an der Einschleuslast gegeben. Die Anzahl der Rundläufer steht in einem funktionalen Zusammenhang zur Anzahl der Endstellen und zum Ausschleusabstand. In den Betrachtungen wird von einem stationären Zustand des Sortersystems ausgegangen. Auf dem Verteilförderer stellt sich ein konstanter Rundläuferanteil ein. 
Der Sorter besitzt einen Einschleusbereich und, davon räumlich getrennt, einen Ausschleusbereich. Alle Endstellen besitzen die gleiche Zuteilungswahrscheinlichkeit sowie den gleichen Ausschleusabstand für alle Transportgüter.

\subsection{Analytische Bestimmung der Rundläufer}

Für den Ausschleusvorgang an einer Endstelle wurde ein Zustandsmodell aufgestellt. Die Auswertung der Zustandsübergänge liefert für die Wahrscheinlichkeit, dass ein ankommendes Transportgut eine abwurfbereite Ausschleusung antrifft,

$$
P\left(Z_{0}\right)=\frac{1}{1+m \cdot p}
$$

mit

$$
\begin{array}{ll}
m= & \text { Ausschleusabstand, } \\
p= & \text { Zielwahrscheinlichkeit (1/Anzahl Sortierziele). }
\end{array}
$$

Für die Transportströme auf dem Verteilförderer können unter Anwendung dieser Zustandsgleichung folgende Bilanzgleichungen aufgestellt werden

$$
N_{0}^{A}=N_{1}^{A} \cdot P\left(Z_{0}\right)=N_{1}^{A} \cdot \frac{1}{1+m \cdot p}
$$

sowie

$$
N_{2}^{A}=N_{1}^{A}-N_{0}^{A}=N_{1}^{A} \cdot\left(1-\frac{1}{1+m \cdot p}\right)
$$

wobei vereinbart gilt

$N_{I n}=$ Transportgut an der Einschleusung,

$N_{1}=$ Transportgut am Punkt nach der Einschleusung,

$N_{2}=$ Transportgut am Punkt nach der Ausschleusung,

$N^{A}=$ Transportgut mit Ziel A,

$N_{0}=$ Leere Transportplätze nach der Ausschleusung,

$N=$ Transportkapazität des Verteilförderers.

Mit den Ansätzen für die Zielwahrscheinlichkeit

$$
p=\frac{N_{1}^{A}}{N}
$$

und die partielle Auslastung

$$
\rho_{A}=\frac{N_{I n}^{A}}{N}
$$

ergibt sich für die Anzahl Rundläufer an einer Endstelle

$$
N_{2}^{A}=N_{I n}^{A} \cdot\left(\frac{m \cdot \rho_{A}}{1-m \cdot \rho_{A}}\right)
$$

und damit für das Verhältnis aller Rundläufer zur gesamten Einschleuslast

$$
\frac{N_{R L}}{N_{I n}}=\frac{m \cdot p \cdot \rho}{1-m \cdot p \cdot \rho}
$$

sowie der Zusammenhang

$$
\rho_{R L}=\frac{N_{R L}}{N}=\frac{m \cdot p \cdot \rho^{2}}{1-m \cdot p \cdot \rho}
$$

für das Verhältnis von Rundläuferanzahl zu Transportkapazität des Verteilförderers.

Der Wert $\rho_{\mathrm{RL}}$ gibt ein Maß für die zusätzliche Belastung des Sorters durch Rundläufer im Ausschleusbereich. 


\subsection{Experimentelle Bestimmung der Rundläufer}

Für die experimentelle Bestimmung der Rundläuferanzahl wurden zahlreiche Simulationsexperimente in einem Parameterraum durchgeführt. Der Ausschleusabstand wurde zwischen 1, 2, 3 und 4 verändert. Die Zahl der Sortierziele wechselte zwischen 12, 18, 24 und 30 in den Experimenten. Die Transportkapazität betrug 7200 Stück pro Stunde. Die Einschleuslast wurde schrittweise von 3000 Stück pro Stunde bis auf 6300 Stück pro Stunde erhöht, soweit es die Gesamtauslastung auf dem Verteilförderer zuließ. Abbildung 2 zeigt den Parameterraum der Simulationsexperimente.

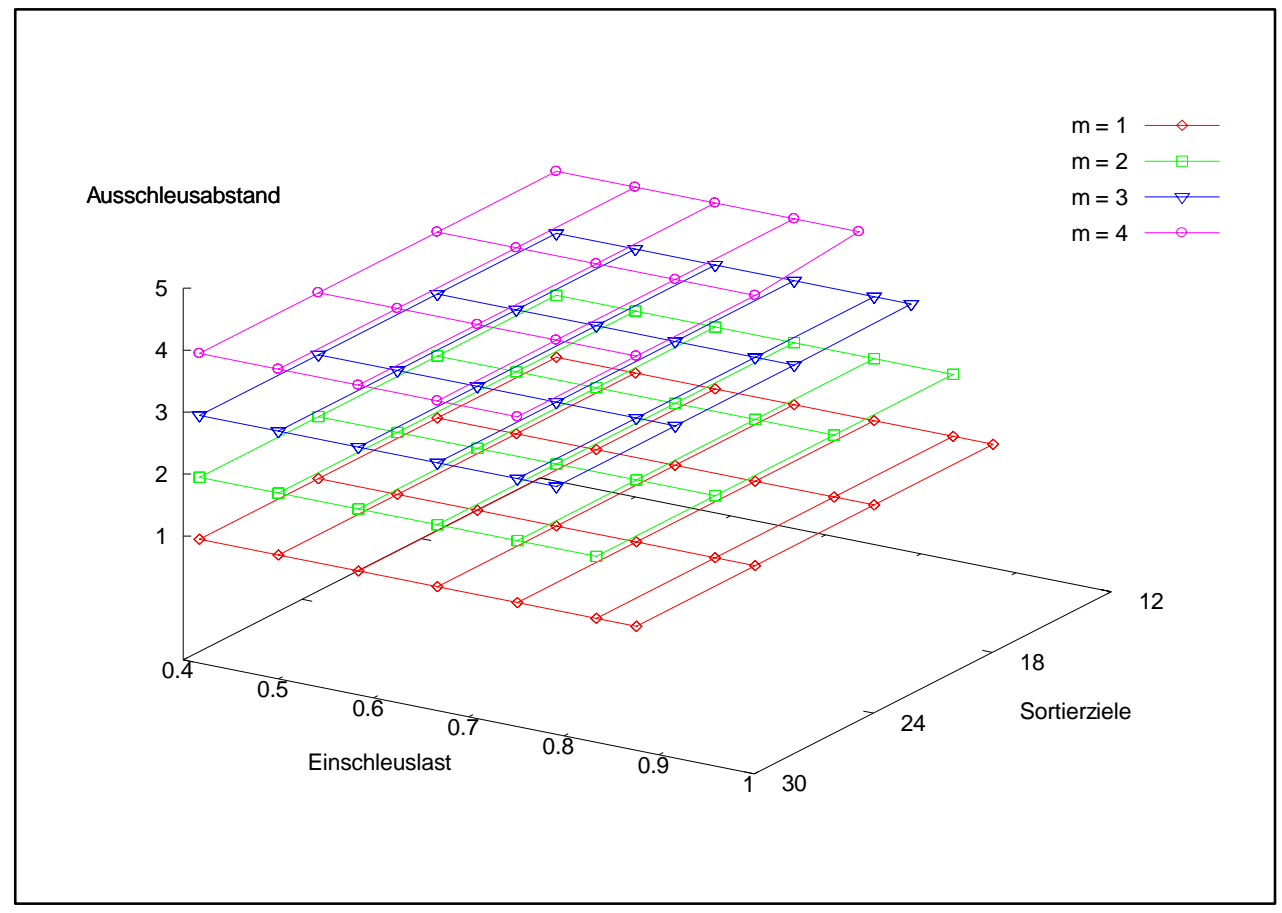

Abbildung 2: Parameterraum Simulation Rundläufer

Im Ergebnis wurde die Anzahl der mehrfach am Einschleusbereich vorbei fahrenden Transportgüter gezählt. Diese Rundläufer ergeben die zusätzliche Belastung des Sorters.

Der Vergleich von theoretischem Ansatz in Gleichung (5) und Simulationsexperimenten zeigt einen relativen Fehler von bis zu 25\%. Der Fehler sinkt tendenziell mit steigender Einschleuslast und mit zunehmender Anzahl der Sortierziele, wobei sich insgesamt ein funktional schwer abzubildender Zusammenhang zwischen dem Fehler und den Experimentparametern zeigt.

Einen alternativen Weg für die Bestimmung der Kenngrößenzusammenhängen bietet auch in diesem Anwendungsfall das Näherungsverfahren Approx auf der Basis von Polynomgleichungen. Für den Wert $\rho_{\mathrm{RL}}$ soll eine Näherung in Abhängigkeit von den Kenngrößen Ausschleusabstand, Anzahl Sortierziele und Auslastung des Einschleusbereichs bestimmt werden.

Tabelle 7: Kenngrößen im Näherungspolynom für $\rho R L$

\begin{tabular}{rc}
\hline Index & Kenngröße \\
\hline 1 & Ausschleusabstand m \\
2 & Anzahl Sortierziele 1/p \\
3 & Auslastung des Einschleusbereichs $\rho$ \\
\hline
\end{tabular}

Die Anzahl der Polynomglieder und der Wertebereich der Exponenten wurde in mehreren Testläufen manuell verändert mit dem Ziel, Anzahl und Wertebereich so klein wie möglich und so groß wie nötig festzulegen. Eine Reduzierung von Anzahl und Wertebereich kann durch die Erhöhung der Prägnanz der hauptsächlichen Zusammenhänge zu einer besseren Akzeptanz der Polynomnäherung in der Praxis führen. 
Die Belegung der Transportplätze durch Rundläufer erreicht im Experiment mit Einschleusabstand $\mathrm{m}=4$ und 12 Sortierzielen einen Anteil von 25\% als maximalen Wert. Der Anteil der Rundläufer im Experiment nimmt zu bei steigenden Ausschleusabstand und bei wachsender Einschleuslast. Die folgende Tabelle zeigt exemplarisch Simulationsergebnisse für einige Parameterkombinationen.

Tabelle 8: Rundläuferresultate

\begin{tabular}{|c|c|c|c|c|}
\hline $\begin{array}{c}\text { Ausschleus- } \\
\text { abstand }\end{array}$ & $\begin{array}{c}\text { Sortier- } \\
\text { ziele }\end{array}$ & $\begin{array}{c}\text { Einschleuslast in } \\
\text { St./h }\end{array}$ & $\begin{array}{c}\text { Rundläufer } \\
\text { pro Stunde }\end{array}$ & $\begin{array}{c}\text { Rundläufer- } \\
\text { zusatzlast }^{1}\end{array}$ \\
\hline 1 & 30 & 5400 & 150 & $2,1 \%$ \\
\hline 2 & 24 & 5400 & 388 & $5,4 \%$ \\
\hline 3 & 18 & 3600 & 285 & $3,9 \%$ \\
\hline 4 & 12 & 3600 & 860 & $12 \%$ \\
\hline
\end{tabular}

Im folgenden werden 3 Näherung mit unterschiedlicher Anzahl der Polynomglieder und unterschiedlicher Näherungsgüte vorgestellt.

$$
\begin{aligned}
& \widetilde{\rho}_{3}=1,3240437657 \cdot m^{2} \cdot p^{2} \cdot \rho^{2}+1,0017005415 \cdot m \cdot p \cdot \rho^{2}+0,0016178638 \\
& \widetilde{\rho}_{2}=1,2136702325 \cdot m^{2} \cdot p^{2} \cdot \rho^{2}+1,0459971582 \cdot m \cdot p \cdot \rho^{2} \\
& \widetilde{\rho}_{1}=1,4034603731 \cdot m^{2} \cdot p^{2} \cdot \rho^{2}+1,0 \cdot m \cdot p \cdot \rho^{2}
\end{aligned}
$$

Der Wert des Koeffizienten $c_{1}$ in $\widetilde{\rho}_{1}$ kann durch den Ausdruck $\sqrt{2}$ angenährt werden. Somit kann man für die Näherung von $\widetilde{\rho}$ auch die Gleichung angeben:

$$
\widetilde{\rho}_{0}=\sqrt{2} \cdot(m \cdot p \cdot \rho)+m \cdot p \cdot \rho^{2}
$$

Tabelle 9: Vergleich der Polynomnäherungen

\begin{tabular}{rrr}
\hline$K$ & $\begin{array}{c}\text { max. rel. } \\
\text { Fehler }\end{array}$ & $\begin{array}{c}\text { max. abs. } \\
\text { Fehler }\end{array}$ \\
\hline 3 & $9,7 \%$ & $38 \mathrm{St} . / \mathrm{h}$ \\
2 & $17,7 \%$ & $29 \mathrm{St} / \mathrm{h}$ \\
1 & $20,7 \%$ & $37 \mathrm{St} . / \mathrm{h}$ \\
\hline
\end{tabular}

Der relative Fehler bezieht sich auf den Zusatzbelastungswert $\rho_{\mathrm{RL}}$ durch Rundläufer. Der absolute Fehler gibt Auskunft über den maximalen Differenzbetrag der resultierenden Rundläuferanzahl in Stück pro Stunde. Die hohen Werte im relativen Fehler treten vorrangig bei den weniger interessanten geringen Absolutwerten auf. Die Ungenauigkeit der Näherung wird dadurch etwas abgeschwächt.

Im Ausschleusbereich können durch einen Ausschleusabstand Rundläufer auf dem Verteilförderer entstehen. Diese zirkulierenden Güter behindern das Einschleusen neuer Güter und verringern dadurch die effektive Sortierleistung. Im Forschungsprojekt wurde eine Näherung für die Berechnung die Rundläuferanzahl und die Leistungsminderung in Abhängigkeit vom Ausschleusabstand bestimmt.

\footnotetext{
${ }^{1}$ bezogen auf die Transportnennleistung des Sorters 


\section{Literatur}

[Beumer1993] Beumer, Christoph: Computerunterstützte Materialflußplanung für Warenverteilsysteme. Düsseldorf: VDI-Verlag 1993 (Fortschritt-Berichte VDI, Reihe 13, Nr. 40) - zugleich Universität Hannover, Dissertation, 1993.

[Hirsch2004]

[JoHo2006]

[MeiSun2007] Hirschmüller, Thomas: Leistungskriterien und Systemfindung für die Stückguteinschleusung auf Sorter. Universität Dortmund, Dissertation, 2004. Jodin, Dirk; Ten Hompel, Michael: Sortier- und Verteilsysteme. Springer Verlag Berlin Heidelberg 2006.

Meinhardt, Ingolf; Sunarjo, Felisia; Marquardt, Hans-Georg: Dimensionierung von Entkopplungspuffern in dynamischen Fertigungsprozessen mittels Warteschlangen. Beitrag zum 3. Fachkolloquium der WGTL, Hamburg, 2007.

[Radtke2000] Radtke, Axel: Beitrag zur Entwicklung optimierter Betriebsstrategien für Sortiersysteme. Dortmund: Praxiswissen, 2000. - zugleich: Universität Dortmund, Dissertation, 2000.

[Schulze2000] Schulze, Frank: Neue Methoden zur Modellbildung für die Planung und Steuerung von Materialflußsystemen. Technische Universität Dresden, Dissertation, 2000. 